\title{
@creative
}

Also available at http://amc-journal.eu

ISSN 1855-3966 (printed edn.), ISSN 1855-3974 (electronic edn.)

ARS MATHEMATICA CONTEMPORANEA 10 (2016) 19-29

\section{Squashing maximum packings of 6-cycles into maximum packings of triples}

\author{
Curt C. Lindner \\ Department of Mathematics and Statistics, Auburn University, \\ Auburn, AL 36849-5307, U.S.A. \\ Giovanni Lo Faro * \\ Dipartimento di Matematica e Informatica, Università di Messina, \\ 98166 Messina, Italia \\ Antoinette Tripodi ${ }^{\dagger}$ \\ Dipartimento di Matematica e Informatica, Università di Messina, \\ 98166 Messina, Italia
}

Received 21 July 2014, accepted 28 September 2014, published online 24 January 2015

\begin{abstract}
A 6-cycle is said to be squashed if we identify a pair of opposite vertices and name one of them with the other (and thereby turning the 6 -cycle into a pair of triples with a common vertex). The squashing problem for 6 -cycle systems was introduced by C. C. Lindner, M. Meszka and A. Rosa and completely solved by determining the spectrum. In this paper, by employing PBD and GDD-constructions and filling techniques, we extend this result by squashing maximum packings of $K_{n}$ with 6-cycles into maximum packings of $K_{n}$ with triples. More specifically, we establish that for each $n \geq 6$, there is a max packing of $K_{n}$ with 6 -cycles that can be squashed into a maximum packing of $K_{n}$ with triples.
\end{abstract}

Keywords: Maximum packing with triples, maximum packing with 6-cycles.

Math. Subj. Class.: 05B07

\footnotetext{
* Supported by P.R.I.N., P.R.A. and I.N.D.A.M.(G.N.S.A.G.A.)

${ }^{\dagger}$ Supported by P.R.I.N., P.R.A. and I.N.D.A.M.(G.N.S.A.G.A.)

E-mail addresses: lindncc@auburn.edu (Curt C. Lindner), lofaro@unime.it (Giovanni Lo Faro), atripodi@unime.it (Antoinette Tripodi)
} 


\section{Introduction}

Let $G$ be a graph. A $G$-design of order $n$ is a pair $(S, B)$ where $B$ is a collection of subgraphs (blocks), each isomorphic to $G$, which partitions the edge set of the complete undirected graph $K_{n}$ with vertex set $S$. After determining the spectrum for $G$-designs for different graphs $G$, many problems have been studied also recently (for example, see [1]-[7]).

A Steiner triple system (more simply, triple system) of order $n$ is a $G$-design of order $n$ where $G$ is the graph $K_{3}$. It is well known that the spectrum for triple systems is precisely the set of all $n \equiv 1$ or $3(\bmod 6)$ [9], and that if $(S, T)$ is a triple system of order $n$, then $|T|=n(n-1) / 6$. Similarly, a 6 -cycle system of order $n$ is a $G$-design of order $n$ where $G$ is 6 -cycle. The spectrum for 6 -cycle systems is precisely the set of all $n \equiv 1$ or 9 (mod 12) [15], and if $(X, C)$ is a 6 -cycle system of order $n$, then $|C|=n(n-1) / 12$. It is worth noting that if $(S, T)$ and $(X, C)$ have order $n$, then $|T|=2|C|$.

Given the fact that triple systems and 6-cycle systems coexist for all $n \equiv 1$ or 9 (mod 12), an obvious question to ask is: are there any connections between the two when $n \equiv$ 1 or $9(\bmod 12)$ ? The answer, of course, is yes. One much studied connection is that of 2-perfect 6-cycle systems. A 6-cycle system is 2-perfect provided the collection of triples obtained by replacing each 6 -cycle $(a, b, c, d, e, f)$ with the two triples $(a, c, e)$ and $(b, d, f)$ is a Steiner triple system. Such systems exist for all $n \equiv 1$ or $9(\bmod 12) \geq 13$ [15].

Quite recently a new connection between triple systems and 6-cycle systems has been introduced: the squashing of a 6-cycle system into a Steiner triple system. A definition is in order. Let $(a, b, c, d, e, f)$ be a 6 -cycle and form the following six bowties (a pair of triples with a common vertex).

If $B$ is any one of the six bowties in Figure 1, we say that we have squashed $(a, b, c, d, e, f)$ into $B$. So there are six different ways to squash a 6 -cycle into a bowtie. If $(X, C)$ is a 6 -cycle system, $2|C|=2 n(n-1) / 12=n(n-1) / 6$ is the number of triples in a Steiner triple system. Therefore it makes sense to ask the following question: what is the spectrum for 6-cycle systems that can be squashed into Steiner triple systems? In [11], a complete solution is given to this problem by constructing for every $n \equiv 1$ or $9(\bmod 12)$ a 6 -cycle system that can be squashed into a Steiner triple system.

\section{Example 1.1. (A 6-cycle system of order 9 squashed into a triple system [11].)}

$\begin{array}{ccc}(0,1,2,3,4,5) & & (0,1,2)(0,4,5) \\ (3,6,0,2,4,1) & & (3,6,0)(3,4,1) \\ (2,8,4,0,3,7) & \text { SQUASH } & (2,8,4)(2,3,7) \\ (7,0,8,6,5,1) & \longrightarrow & (7,0,8)(7,5,1) \\ (6,1,8,5,7,4) & & (6,1,8)(6,7,4) \\ (5,2,6,7,8,3) & & (5,2,6)(5,8,3)\end{array}$

Now if $n \equiv 3$ or $7(\bmod 12)$ there does not exist a 6 -cycle system of order $n$. However, there does exist a maximum packing (max packing) of $K_{n}$ with 6 -cycles with leave a triple (i.e., a pair $(X, C)$ and a set $L$, the leave, where $C$ is a collection of edge disjoint 6 -cycles with verteces in $X, L$ is the set of the edges of $K_{n}$ not belonging to any 6 -cycle of $C$ and $|L|$ is as small as possible) and so the following question makes sense. Does there exist for each $n \equiv 3$ or $7(\bmod 12)$ a max packing of $K_{n}$ with 6 -cycles which can be squashed into bowties so that the bowties plus the leave (a triple) form a Steiner triple system? 

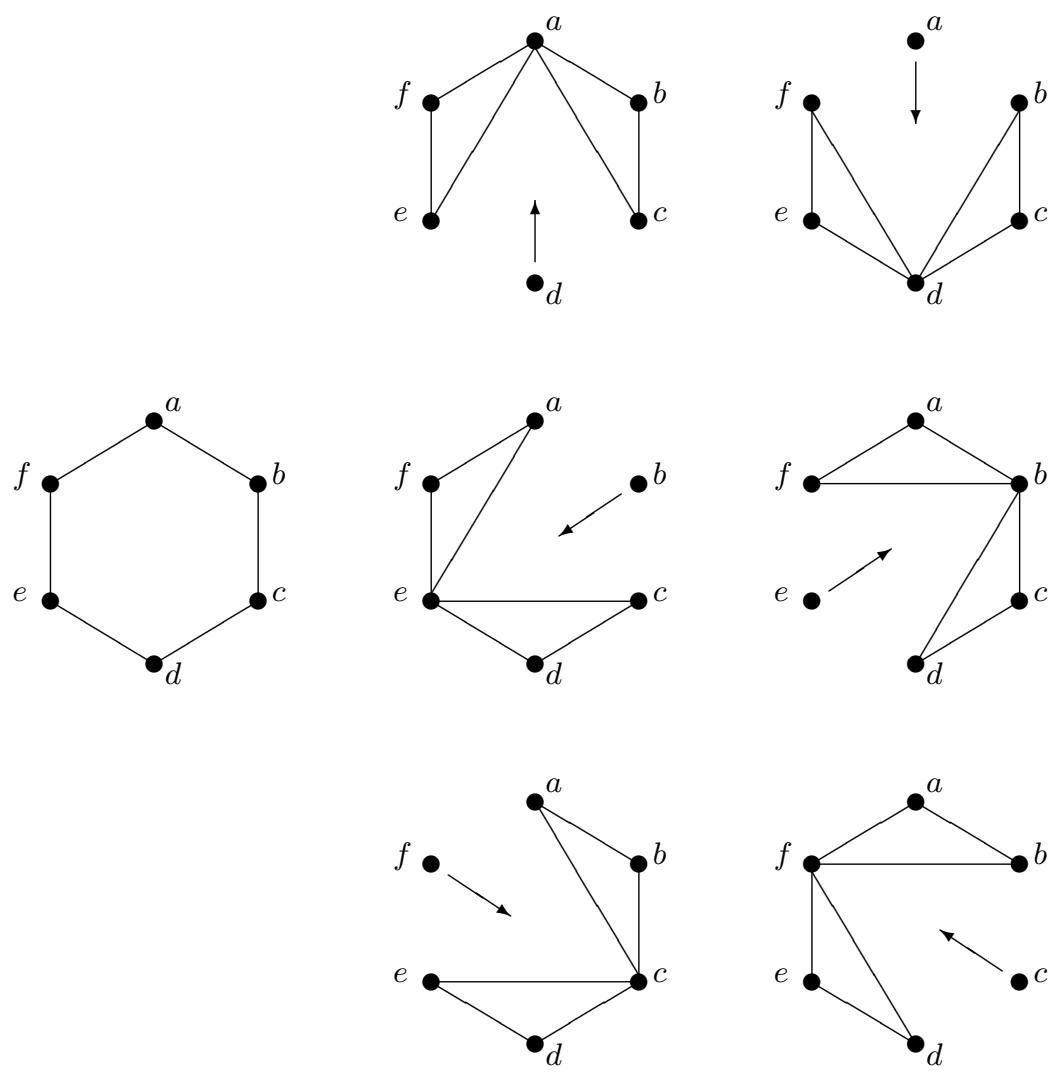

Figure 1

Example 1.2. (A max packing of $K_{7}$ squashed into a triple system [11].)

$$
\begin{array}{ccc}
(2,3,4,5,0,1) & \text { SQUASH } & (2,1,0)(2,3,4) \\
(4,6,0,2,5,1) & \longrightarrow & (4,1,5)(4,0,6) \\
(5,6,2,4,0,3) & & (5,3,0)(5,6,2) \\
(1,3,6) \text { leave } & \longrightarrow & (1,3,6)
\end{array}
$$

The following theorem is proved in [11].

Theorem 1.3. [11] There exists a 6 -cycle system of every order $n \equiv 1$ or $9(\bmod 12)$ that can be squashed into a triple system and a 6-cycle maximum packing that can be squashed into a triple system for every $n \equiv 3$ or $7(\bmod 12), n \geq 7$.

The object of this paper is to finish off the problem of squashing maximum packings of $K_{n}$ with 6-cycles into maximum packings of $K_{n}$ with triples. We need to be a bit more precise.

Let $(X, C)$ be a maximum packing of $K_{n}$ with 6 -cycles with leave $L$. In what follows, to keep the vernacular from getting out of hand, to say that $C$ has been squashed means that the resulting collection $S(C)$ of bowties is a partial triple system.

Further, if $t$ is a triple belonging to $L$ and $S(C) \cup\{t\}$ is a maximum packing of $K_{n}$ with triples (or a triple system), we will say that we have squashed $(X, C)$ into a maximum 
packing of $K_{n}$ with triples. So, for example, Example 1.2 is the squashing of a maximum packing of $K_{7}$ with 6-cycles into a triple system of order 7 .

The following easy to read table gives the leaves for max packings for both 6 -cycles and triples not covered by Theorem 1.3. (See [8] and [13].)

\begin{tabular}{|c|c|c|}
\hline$K_{n}$ & 6-cycles leave & triples leave \\
\hline \hline$n \equiv 0,2,6,8(\bmod 12)$ & 1 -factor & 1-factor \\
\hline$n \equiv 5(\bmod 12)$ & 4 -cycle & 4-cycle \\
\hline$n \equiv 11(\bmod 12)$ & 4 leaves are possible & 22 leaves are \\
$n \equiv 4$ or $10(\bmod 12)$ & possible for $n \geq 16$ & $\bullet$
\end{tabular}

We remark that if $n \equiv 0,2,6,8$ or $5(\bmod 12)$ and a 6 -cycle maximum packing can be squashed, there are no triples to be added; i.e., the resulting collection of bowties is a maximum packing of $K_{n}$ with triples. If $n \equiv 4,10$ or $11(\bmod 12)$ and a 6 -cycle maximum packing can be squashed, then a triple is taken from the 6 -cycle leave in order to obtain a maximum packing of $K_{n}$ with triples.

\section{Preliminaries}

From now on to say that the 6 -cycle $(a, b, c, d, e, f)$ is squashed we will always mean that it has been squashed into the bowtie $(a, b, c)(a, e, f)$; see Figure 2.
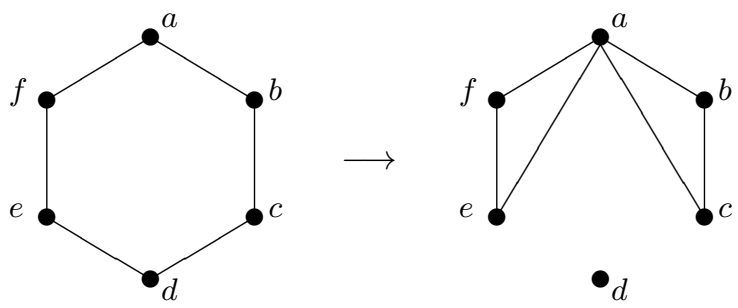

Figure 2

So, for example, in Example 1.1 we can simply list the 6-cycles (without listing the bowties they have been squashed into) and say they can be squashed into a triple system.

The following three examples are used repeatedly in what follows.

Example 2.1. (A max packing of $K_{6}$ with 6-cycles squashed into a max packing of $K_{6}$ with triples.)

$C=\{(5,0,1,2,4,3),(2,3,1,5,4,0)\}$, leave $L=\{(0,3),(1,4),(2,5)\}$. (There are no triples in the leave.) 
Example 2.2. (A max packing of $K_{8}$ with 6-cycles squashed into a max packing of $K_{8}$ with triples.)

$X=Z_{4} \times Z_{2} ; C=\left\{\left(0_{0}, 3_{0}, 1_{1}, 2_{0}, 3_{1}, 0_{1}\right),\left(1_{0}, 0_{0}, 2_{1}, 3_{0}, 0_{1}, 1_{1}\right),\left(2_{0}, 1_{0}, 3_{1}, 0_{0}, 1_{1}, 2_{1}\right)\right.$, $\left.\left(3_{0}, 2_{0}, 0_{1}, 1_{0}, 2_{1}, 3_{1}\right)\right\}$, leave $L=\left\{\left(0_{0}, 2_{0}\right),\left(1_{0}, 3_{0}\right),\left(0_{1}, 2_{1}\right),\left(1_{1}, 3_{1}\right)\right\}$. (There are no triples in the leave.)

Example 2.3. (Decomposition of $K_{4,4,4}$ into 6-cycles squashed into triples.) (An obvious definition.)

$X=Z_{4} \times\{1,2,3\} ; C=\left\{\left(1_{2}, 1_{3}, 0_{1}, 0_{2}, 0_{3}, 1_{1}\right),\left(0_{2}, 2_{3}, 0_{1}, 1_{2}, 0_{3}, 2_{1}\right),\left(1_{1}, 0_{2}, 3_{3}, 0_{1}, 2_{2}\right.\right.$, $\left.1_{3}\right),\left(0_{1}, 3_{2}, 3_{3}, 1_{1}, 2_{2}, 0_{3}\right),\left(3_{2}, 1_{1}, 2_{3}, 1_{2}, 3_{1}, 0_{3}\right),\left(1_{2}, 2_{1}, 2_{3}, 3_{2}, 3_{1}, 3_{3}\right),\left(3_{1}, 0_{2}, 1_{3}, 2_{1}\right.$, $\left.\left.2_{2}, 2_{3}\right),\left(2_{1}, 3_{2}, 1_{3}, 3_{1}, 2_{2}, 3_{3}\right)\right\}$. (There is no leave.)

\section{Basic Lemmas}

With the examples of Section 2 in hand we can go to the general constructions, where we shall make use of GDDs. Let $H$ be a set of integers and $X$ be a set of size $n$; a $\operatorname{GDD}(n, H, k)$ is a triple $(X, G, B)$ where $G$ is a partition of $X$ into subsets called groups of size in $H, B$ is a set of subsets of $X$ (called blocks) of size $k$, such that a group and a block contain at most one common point and every pair of points from distinct groups occurs in exactly one block. A PBD is a $\operatorname{GDD}(n,\{1\}, k)$.

We break the constructions into the eight cases: $2,6,8 ; 0 ; 11 ; 4,10 ; 5(\bmod 12)$.

\section{$3.1 n \equiv 2,6$ and $8(\bmod 12)$}

These are the easiest cases, so a good place to start.

$\underline{n \equiv 2(\bmod 12)} \quad$ Write $12 k+2=2(6 k+1)$ and let $(X, T)$ be a Steiner triple system of order $6 k+1$. Let $S=X \times\{1,2\}$ and define a collection $C$ of 6 -cycles as follows: For each triple $t=\{a, b, c\} \in T$ define a copy of Example 2.1 on $\{a, b, c\} \times\{1,2\}$ with leave $L_{t}=\left\{\left(a_{1}, a_{2}\right),\left(b_{1}, b_{2}\right),\left(c_{1}, c_{2}\right)\right\}$ and put these 6 -cycles in $C$. Then $C$ is a max packing of $K_{12 k+2}$ with 6 -cycles with leave $L=\left\{L_{t} \mid t \in T\right\}$. Trivially, $C$ can be squashed into a max packing of $K_{12 k+2}$ with triples with leave $L$.

$n \equiv 6(\bmod 12) \quad$ The case for $n=6$ is handled with Example 2.1. So now write $12 k+6=$

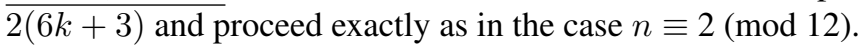

$n \equiv 8(\bmod 12) \quad$ Write $12 k+8=2(6 k+4)$. The case $n=8$ is handled by Example 2.2. So let $12 k+8 \geq 20$. It is well kown that there is a PBD with block sizes 3 and 4 for every $n \equiv 4(\bmod 6)[13]$. Let $(X, B)$ be such a PBD, $|X| \equiv 4(\bmod 6)$, and proceed exactly as in the cases for $n \equiv 2$ or $6(\bmod 12)$, using Example 2.2 as well as Example 2.1.

Lemma 3.1. There exists a max packing of $K_{n}$ with 6-cycles that can be squashed into a max packing of $K_{n}$ with triples for all $n \equiv 2,6,8(\bmod 12) \geq 6$.

\section{$3.2 n \equiv 0(\bmod 12)$}

We begin with an example.

Example 3.2. $(n=12)$

Let $X=\left\{\infty_{1}, \infty_{2}\right\} \cup Z_{10}$ and define a collection of 6 -cycles $C$ as follows: 


$$
\begin{array}{llll}
\left(0, \infty_{1}, 2,1,3,6\right), & \left(2, \infty_{2}, 4,3,5,8\right), & \left(4, \infty_{1}, 6,5,7,0\right), & \left(6, \infty_{2}, 8,7,9,2\right) \\
\left(8, \infty_{1}, 9,0,1,4\right), & \left(1, \infty_{1}, 3,0,2,7\right), & \left(3, \infty_{2}, 5,2,4,9\right), & \left(5, \infty_{1}, 7,4,6,1\right), \\
\left(7, \infty_{2}, 9,6,8,3\right), & \left(0, \infty_{2}, 1,8,9,5\right), & &
\end{array}
$$

with leave $L=\left\{(0,8),(1,9),(2,3),(4,5),(6,7),\left(\infty_{1}, \infty_{2}\right)\right\}$. Then $(X, C)$ is a max packing of $K_{12}$ with 6 -cycles and can be squashed into a max packing of $K_{12}$ with triples with leave $L$.

We will need two constructions for $12 k \geq 24$ : one when $k$ is even and one when $k$ is odd.

$\underline{12 k, k \text { even }}$ Write $12 k=4(3 k)$ and let $(P, G, B)$ be a $\operatorname{GDD}(3 k,\{2\}, 3)$, set $X=$ $\overline{P \times\{1,2,3}, 4\}$ and define a collection of 6 -cycles $C$ as follows:

(i) For each group $g \in G$ place Example 2.2 on $g \times\{1,2,3,4\}$ with leave $L_{g}=\{g \times$ $\{1\}, g \times\{2\}, g \times\{3\}, g \times\{4\}\}$ and place these 6 -cycles in $C$.

(ii) For each triple $t=\{a, b, c\} \in B$ place a copy of Example 2.3 on $K_{4,4,4}$ with parts $\{a\} \times\{1,2,3,4\},\{b\} \times\{1,2,3,4\}$, and $\{c\} \times\{1,2,3,4\}$ and place these 6 -cycles in $C$.

Then $(X, C)$ is a max packing of $K_{12 k}$ with 6 -cycles with leave $L=\{g \times\{1\}, g \times\{2\}, g \times$ $\{3\}, g \times\{4\} \mid g \in G\}$. It is straightforward to see that the 6 -cycles in (i) and (ii) can be squashed into a max packing of $K_{12 k}$ with triples with leave $L$.

$12 k, k$ odd Write $12 k=4(3 k)$. Since $k$ is odd, $3 k$ is the order of a Kirkman triple system $\overline{(P, T) \text {. Let }} X=P \times\{1,2,3,4\}, \pi$ a parallel class in $T$, and define a collection of 6 -cycles $C$ as follows:

(i) For each triple $t=\{a, b, c\} \in \pi$, place a copy of Example 3.2 on $\{a, b, c\} \times$ $\{1,2,3,4\}$ with leave $L_{t}$ and place these 6 -cycles in $C$.

(ii) For each triple $\{a, b, c\} \in T \backslash \pi$, place a copy of Example 2.3 on $K_{4,4,4}$ with parts $\{a\} \times\{1,2,3,4\},\{b\} \times\{1,2,3,4\}$, and $\{c\} \times\{1,2,3,4\}$, and place these 6 -cycles in $C$.

Then $(X, C)$ is a max packing of $K_{12 k}$ with 6 -cycles with leave $L=\left\{L_{t} \mid t \in \pi\right\}$. Squashing these 6 -cycles produces a max packing of $K_{12 k}$ with triples with leave $L$.

Lemma 3.3. There exists a max packing of $K_{n}$ with 6-cycles that can be squashed into a max packing of $K_{n}$ with triples for all $n \equiv 0(\bmod 12)$.

\section{$3.3 n \equiv 11(\bmod 12)$}

We begin with an example.

Example 3.4. $(n=11)$

Let $X=Z_{9} \cup\left\{\infty_{1}, \infty_{2}\right\}$ and define a collection of 6 -cycles $C$ as follows:
$\left(4,8, \infty_{2}, 7, \infty_{1}, 0\right)$,
$\left(5,0, \infty_{2}, 4, \infty_{1}, 1\right)$
$\left(6,1, \infty_{2}, 5, \infty_{1}, 2\right)$,
$\left(7,2, \infty_{2}, 6, \infty_{1}, 3\right)$
$(3,2,5,7,0,1)$,
$(7,4,5,3,0,6)$
$(4,3,6,8,1,2)$,
$(8,5,6,4,1,7)$ 
with leave $L=\left\{\left(\infty_{1}, \infty_{2}, 3,8\right),(0,2,8)\right\}$. Then $(X, C)$ is a max packing of $K_{11}$ with 6 -cycles with leave L. Squashing these 6 -cycles and adding $(0,2,8)$ from the leave L gives a max packing of $K_{11}$ with triples with leave the 4-cycle $\left(\infty_{1}, \infty_{2}, 3,8\right)$.

We can now give a general construction for $11(\bmod 12) \geq 23$.

$12 k+11 \geq 23 \quad$ Write $12 k+11=2(6 k+4)+3$. Let $(P, G, B)$ be a $\operatorname{GDD}(6 k+$ $\overline{\left.4,\left\{4^{*}, 2\right\}, 3\right)[13]}$, set $X=\left\{\infty_{1}, \infty_{2}, \infty_{3}\right\} \cup(P \times\{1,2\})$, and define a collection of 6 -cycles $C$ as follows:

(i) Let $b^{*}$ be the unique group of size 4 and define a copy of Example 3.4 on $\left\{\infty_{1}, \infty_{2}\right.$, $\left.\infty_{3}\right\} \cup\left(b^{*} \times\{1,2\}\right)$ with leave $L=\left\{\left(\infty_{1}, \infty_{2}, \infty_{3}\right),(x, y, z, w)\right\}$, where $\{x, y, z, w\}$ $\subseteq b^{*} \times\{1,2\}$ and place these 6 -cycles in $C$.

(ii) For each group $g \in G$ of size 2, define a copy of a max packing of $K_{7}$ with 6 -cycles, with vertex set $\left\{\infty_{1}, \infty_{2}, \infty_{3}\right\} \cup(b \times\{1,2\})$, that can be squashed into 6 -triples with leave $\left(\infty_{1}, \infty_{2}, \infty_{3}\right)$ [11]. Add these 6 -cycles to $C$.

(iii) For each triple $t=\{a, b, c\} \in B$, place a copy of Example 2.1 on $t \times\{1,2\}$ with leave $\{a\} \times\{1,2\},\{b\} \times\{1,2\}$, and $\{c\} \times\{1,2\}$ and place these 6 -cycles in $C$.

Then $(X, C)$ is a max packing of $K_{12 k+11}$ with 6 -cycles with leave $L$ in (i). If we squash these 6 -cycles and add the triple $\left(\infty_{1}, \infty_{2}, \infty_{3}\right)$ from the leave $L$ in (i) we have a max packing of $K_{12 k+11}$ with triples with leave $(x, y, z, w)$ in (i).

Lemma 3.5. There exists a max packing of $K_{n}$ with 6 -cycles that can be squashed into a max packing of $K_{n}$ with triples for all $n \equiv 11(\bmod 12)$.

\section{$3.4 n \equiv 4$ or $10(\bmod 12)$}

The following three examples are necessary for the constructions in this section.

Example 3.6. $(n=10)$

Let $X=\{\infty\} \cup\left(Z_{3} \times Z_{3}\right)$ and define a collection of 6 -cycles $C$ as follows: $\left(0_{1}, 1_{1}, 0_{0}, 1_{2}\right.$, $\left.0_{2}, \infty\right),\left(1_{2}, 2_{1}, 0_{0}, 0_{1}, 0_{2}, 1_{0}\right),\left(1_{1}, 2_{1}, 1_{0}, 2_{2}, 1_{2}, \infty\right),\left(2_{2}, 0_{1}, 1_{0}, 1_{1}, 1_{2}, 2_{0}\right),\left(2_{1}, 0_{1}, 2_{0}\right.$, $\left.0_{2}, 2_{2}, \infty\right),\left(0_{2}, 1_{1}, 2_{0}, 2_{1}, 2_{2}, 0_{0}\right)$ with leave $L=\left\{\left\{\infty, 2_{0}, 1_{0}, 0_{0}\right\},\left(0_{1}, 1_{2}\right),\left(1_{1}, 2_{2}\right)\right.$, $\left.\left(2_{1}, 0_{2}\right)\right\}$. (We remark that $\left\{\infty, 2_{0}, 1_{0}, 0_{0}\right\}$ is a copy of $K_{4}$ and not a 4-cycle.) Then $(X, C)$ is a max packing of $K_{10}$ with 6 -cycles with leave $L$. If we squash these 6 -cycles and remove a triple from $\left\{\infty, 2_{0}, 1_{0}, 0_{0}\right\}$, the result is a max packing of $K_{10}$ with triples with leave the tripole $K_{1,3} \cup\left\{\left(0_{1}, 1_{2}\right),\left(1_{1}, 2_{2}\right),\left(2_{1}, 0_{2}\right)\right\}$.

Example 3.7. $(n=16)$

Let $X=\left\{\infty_{1}, \infty_{2}, \infty_{3}, \infty_{4}\right\} \cup\left\{i_{j} \mid i \in Z_{6}, j \in\{0,1\}\right\}$. Further, for each $i \in Z_{6}$, define $\alpha(i)=\infty_{1}$ if $i$ is odd and $\infty_{2}$ if $i$ is even. For each $i \in Z_{6}$ define a collection of 6 -cycles $C$ as follows: $\left(i_{1}, i_{0},(4+i)_{1},(2+i)_{1},(1+i)_{1}, \alpha(i)\right),\left(i_{0},(1+i)_{1},(4+\right.$ $\left.i)_{0},(2+i)_{0},(1+i)_{0}, \alpha(i)\right)$, and $\left(i_{0},(2+i)_{1}, \infty_{3},(1+i)_{0}, \infty_{4},(5+i)_{1}\right)$ with leave $L=\left\{\infty_{1}, \infty_{2}, \infty_{3}, \infty_{4}\right\} \cup\left\{\left(i_{j},(3+i)_{j}\right) \mid i \in\{0,1,2\}, j \in\{0,1\}\right\}$. (Once again we remark that $\left\{\infty_{1}, \infty_{2}, \infty_{3}, \infty_{4}\right\}$ is a copy of $K_{4}$.) Then $(X, C)$ is a max packing of $K_{16}$ with 6 -cycles with leave $L$. If we squash these 6 -cycles and remove a triple from $\left\{\infty_{1}, \infty_{2}, \infty_{3}, \infty_{4}\right\}$, the result is a max packing of $K_{16}$ with triples, with leave the tripole $K_{1,3} \cup\left\{\left(i_{j},(3+i)_{j}\right) \mid i \in\{0,1,2\}, j \in\{0,1\}\right\}$.

Example 3.8. $(n=28)$ 
Let $X=\left\{\infty_{1}, \infty_{2}, \infty_{3}, \infty_{4}\right\} \cup\left(Z_{12} \times\{1,2\}\right)$, and let $(P, G, B)$ be a $\operatorname{GDD}(12,\{3\}, 4)$ (equivalent to a pair of orthogonal quasigroups of order 3 ) and define a collection of 6 cycles $C$ as follows:

(i) For each group $g \in G$, place a copy of Example 3.6 on $\left\{\infty_{1}, \infty_{2}, \infty_{3}, \infty_{4}\right\} \cup(g \times$ $\{1,2\})$ with leave $\left\{\infty_{1}, \infty_{2}, \infty_{3}, \infty_{4}\right\} \cup\left\{\left(x_{1}, x_{2}\right) \mid x \in g\right\}$ (with $K_{4}$ based on $\left.\left\{\infty_{1}, \infty_{2}, \infty_{3}, \infty_{4}\right\}\right)$.

(ii) For each block $b \in B$ place a copy of Example 2.2 on $b \times\{1,2\}$ with leave $\left\{\left\{x_{1}, x_{2}\right\} \mid x \in b\right\}$.

Then $(X, C)$ is a max packing of $K_{28}$ with 6 -cycles with the leave in (i). Now squashing the 6 -cycles in (i) and (ii) and removing a triple from $K_{4}$ gives a max packing of $K_{28}$ with triples with leave a tripole.

We can now go to the general constructions for $n \equiv 10(\bmod 12), n \geq 22$ and $n \equiv 4$ $(\bmod 12), n \geq 40$.

$n \equiv 10(\bmod 12), n \geq 22 \quad$ Write $12 k+10=2(6 k+5)$ and let $(P, B)$ be a $\operatorname{PBD}(6 k+$

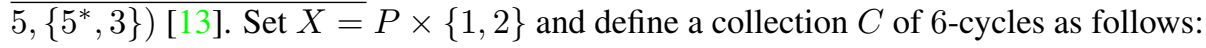

(i) Let $b^{*}$ be the unique block of size 5 and define a copy of Example 3.6 on $b^{*} \times\{1,2\}$ and place these 6-cycles in $C$. (The leave is $K_{4} \cup\{1$-factor $\}$.)

(ii) For each triple $t=\{a, b, c\} \in B$, define a copy of Example 2.1 on $t \times\{1,2\}$ with leave $\left\{\left(a_{1}, a_{2}\right),\left(b_{1}, b_{2}\right),\left(c_{1}, c_{2}\right)\right\}$ and place these 6 -cycles in $C$.

Then $(X, C)$ is a max packing of $K_{12 k+10}$ with 6 -cycles with leave $K_{4} \cup\{1$-factor $\}$. Squashing the 6 -cycles in $C$ and removing a triple from the leave in (i) produces a max packing of $K_{12 k+10}$ with triples with leave a tripole.

$n \equiv 4(\bmod 12), n \geq 40$ Write $12 k+4=4+2(6 k)$ and let $(P, G, B)$ be a $\operatorname{GDD}(6 k,\{6\}$, 3) [13]. Set $X=\left\{\infty_{1}, \infty_{2}, \infty_{3}, \infty_{4}\right\} \cup(P \times\{1,2\})$ and define a collection of 6 -cycles as follows:

(i) For each group $g \in G$ define a copy of Example 3.7 on $\left\{\infty_{1}, \infty_{2}, \infty_{3}, \infty_{4}\right\} \cup(g \times$ $\{1,2\})$ with leave $K_{4} \cup\left\{\left(x_{1}, x_{2}\right) \mid x \in G\right\}\left(K_{4}\right.$ is based on $\left.\left\{\infty_{1}, \infty_{2}, \infty_{3}, \infty_{4}\right\}\right)$.

(ii) For each triple $t=\{a, b, c\} \in B$, define a copy of Example 2.1 on $t \times\{1,2\}$ with leave $\left\{\left(a_{1}, a_{2}\right),\left(b_{1}, b_{2}\right),\left(c_{1}, c_{2}\right)\right\}$ and place these 6 -cycles in $C$.

Then $(X, C)$ is a max packing of $K_{12 k+4}$ with 6 -cycles. Squashing the 6 -cycles in $C$ and removing a triple from the leave $K_{4}$ in (i) produces a max packing of $K_{12 k+4}$ with triples with leave a tripole.

Lemma 3.9. There exists a max packing of $K_{n}$ with 6-cycles that can be squashed into a max packing of $K_{n}$ with triples for all $n \equiv 4$ or $10(\bmod 12) \geq 6$.

\section{$3.5 n \equiv 5(\bmod 12)$}

This case requires three examples.

Example 3.10. $(n=17)$ 
Let $X=\left\{\infty_{1}, \infty_{2}\right\} \cup Z_{15}$ and define a collection of 6 -cycles $C$ as follows: $\left\{(0,9,4,5,1,3)+i \mid i \in Z_{15}\right\} \cup\left\{\left(7,14, \infty_{2}, 13, \infty_{1}, 0\right),\left(8,0, \infty_{2}, 7, \infty_{1}, 1\right),\left(9,1, \infty_{2}, 8\right.\right.$, $\left.\infty_{1}, 2\right),\left(10,2, \infty_{2}, 9, \infty_{1}, 3\right),\left(11,3, \infty_{2}, 10, \infty_{1}, 4\right),\left(12,4, \infty_{2}, 11, \infty_{1}, 5\right),\left(13,5, \infty_{2}\right.$, $\left.\left.12, \infty_{1}, 6\right)\right\}$ with leave the 4 -cycle $\left(\infty_{1}, \infty_{2}, 6,14\right)$. Squashing all of the 6 -cycles in $C$ produces a max packing of $K_{17}$ with triples with leave the 4 -cycle $\left(\infty_{1}, \infty_{2}, 6,14\right)$.

Example 3.11. $(n=29)$

Let $X=\left\{\infty_{1}, \infty_{2}\right\} \cup Z_{27}$ and define a collection of 6 -cycles $C$ as follows:

$\left\{(0,3,1,5,4,9)+i,(0,16,10,17,7,15)+i \mid i \in Z_{27}\right\} \cup\left\{\left(14,0, \infty_{2}, 13, \infty_{1}, 1\right)+\right.$ $\left.j \in\{0,1,2, \ldots, 11\},\left(13,26, \infty_{2}, 25, \infty_{1}, 0\right)\right\}$ with leave the 4 - cycle $\left(\infty_{1}, \infty_{2}, 12,26\right)$. Squashing the 6 -cycles in $C$ gives a max packing of $K_{29}$ with triples with leave the 4-cycle $\left(\infty_{1}, \infty_{2}, 12,26\right)$.

\section{Example 3.12. $(n=53)$}

Let $X=\left\{\infty_{1}, \infty_{2}\right\} \cup Z_{51}$ and define a collection of 6 -cycles $C$ as follows: $\{(0,3,1,5,4,21)+i,(0,19,9,20,11,23)+i,(0,24,8,15,7,22)+i,(0,20,6,11,5,18)+$ $\left.i \mid i \in Z_{51}\right\} \cup\left\{\left(26,0, \infty_{2}, 25, \infty_{1}, 1\right)+j \mid j \in\{0,1,2, \ldots, 23\}\right\} \cup\left\{\left(25,50, \infty_{2}, 49, \infty_{1}\right.\right.$, $0)\}$ with leave the 4 -cycle $\left(\infty_{1}, \infty_{2}, 24,50\right)$. Squashing these 6 -cycles gives a max packing of $K_{53}$ with triples with leave the 4-cycle $\left(\infty_{1}, \infty_{2}, 24,50\right)$.

We can now give two general constructions to finish off the case $n \equiv 5(\bmod 12)$. $12 k+5, k$ odd Write $12 k+5=1+4(3 k+1)$. Since $k$ is odd, $1+4(3 k+1)=1+4(6 t+4)$. $\overline{\text { Let }}(P, G, B)$ be a $\operatorname{GDD}\left(6 t,\left\{4^{*}, 2\right\}, 3\right)$, set $X=\{\infty\} \cup(P \times\{1,2,3,4\})$ and define a collection of 6 -cycles as follows:

(i) For the unique group $b^{*}$ of size 4 , define a copy of Example 3.10 on $\{\infty\} \cup\left(b^{*} \times\right.$ $\{1,2,3,4\}$ ) (the leave is a 4-cycle) and place these 6 -cycles in $C$.

(ii) For each group $g$ of size 2, define a copy of Example 1.1 on $\{\infty\} \cup(g \times\{1,2\})$ and place these 6 -cycles in $C$. (There is no leave.)

(iii) For each triple $\{a, b, c\} \in B$, place a copy of Example 2.3 on $\{a, b, c\} \times\{1,2,3,4\}$ with parts $\{a\} \times\{1,2,3,4\},\{b\} \times\{1,2,3,4\},\{c\} \times\{1,2,3,4\}$, and place these 6 -cycles in $C$. (There is no leave.)

Then $(X, C)$ is a max packing of $K_{12 k+5}$ with 6 -cycles with leave a 4-cycle. If we squash the 6 -cycles in (i), (ii) and (iii), we have a max packing of $K_{12 k+5}$ with triples with leave a 4-cycle.

$12 k+5, k$ even Write $12 k+5=1+4(3 k+1)$. Since $k$ is even, $1+4(3 k+1)=$ $1+4(6 t+1)$. Since $12 k+5 \geq 77,6 t+1 \geq 19$, and there exists a $\operatorname{GDD}\left(6 t+1,\left\{7^{*}, 3\right\}, 3\right)$ [11] $(P, G, B)$. Define a collection $C$ of 6 -cycles on $X=\{\infty\} \cup(P \times\{1,2,3,4\})$ as follows:

(i) For the unique group $b^{*}$ of size 7 , define a copy of Example 3.11 on $\{\infty\} \cup(b \times$ $\{1,2,3,4\}$ ) (with leave a 4-cycle) and place these 6-cycles in $C$.

(ii) For each group $g$ of size 3, place a copy of a 6-cycle system of order 13 which can be squashed into a triple system [11] (no leave) on $\{\infty\} \cup(g \times\{1,2,3,4\})$ and place these 6-cycles in $C$.

(iii) For each triple $\{a, b, c\} \in B$, place a copy of Example 2.3 on $\{a, b, c\} \times\{1,2,3,4\}$ with parts $\{a\} \times\{1,2,3,4\},\{b\} \times\{1,2,3,4\},\{c\} \times\{1,2,3,4\}$, and place these 6 -cycles in $C$. (There is no leave.) 
Then $(X, C)$ is a max packing of $K_{12 k+5}$ with 6 -cycles with leave the 4-cycle in (i). Squashing the 6 -cycles in (i), (ii) and (iii) produces a max packing of $K_{12 k+5}$ with triples with leave the 4-cycle in (i).

Lemma 3.13. There exists a max packing of $K_{n}$ with 6 -cycles that can be squashed into a max packing of $K_{n}$ with triples for all $n \equiv 5(\bmod 12) \geq 17$.

\section{Main result and further developments}

Putting together the results in Section 3 gives the following theorem.

Theorem 4.1. For each $n \equiv 0,2,4,5,6,8,10,11(\bmod 12), n \geq 6$, there exists a max packing of $K_{n}$ with 6-cycles that can be squashed into a max packing of $K_{n}$ with triples. There are no exceptions.

Since a complete solution is also a max packing, we can combine Theorem 1.3 and Theorem 8.1 into the following corollary (giving a complete solution to the squashing of max packings of 6 -cycles into max packings with triples).

Corollary 4.2. For each $n \geq 6$, there is a max packing of $K_{n}$ with 6 -cycles that can be squashed into a max packing of $K_{n}$ with triples.

In this paper we give a complete solution to the problem of squashing maximum packings of $K_{n}$ with 6 -cycles into maximum packings of $K_{n}$ with triples. An open problem is to solve the general case, i.e. squashing a maximum packing of $K_{n}$ with $2 m$-cycles into a maximum packing of $K_{n}$ with $m$-cycles; the case $m=4$ is completely solved in [10] .

\section{References}

[1] L. Berardi, M. Gionfriddo and R. Rota, Perfect octagon quadrangle systems - II, Discrete Math. 312(3) (2012), 614-620.

[2] Y. Chang, T. Feng, G. Lo Faro and A. Tripodi, The existence spectrum of $\operatorname{Meta}\left(K_{4}^{(3)}>K_{4}^{(3)}-\right.$ e), Science China Mathematics 11 (53) (2010), 2865-2876.

[3] Y. Chang, T. Feng, G. Lo Faro and A. Tripodi, The triangle intersection numbers of a pair of disjoint S(2, 4, v)s, Discrete Math. 310(21) (2010), 3007-3017.

[4] Y. Chang, T. Feng, G. Lo Faro and A. Tripodi, The fine triangle intersection for $\left(K_{4}-e\right)$ designs, Discrete Math. 311(21) (2011), 2442-2462.

[5] Y. Chang, T. Feng, G. Lo Faro and A. Tripodi, The fine triangle intersection for kite systems, Discrete Math. 312 (3) (2012), 545-553.

[6] Y. Chang, G. Lo Faro and A. Tripodi, Tight blocking sets in some maximum packings of $\lambda K_{n}$, Discrete Math. 308 (2008), 427-438.

[7] Y. Chang, G. Lo Faro and A. Tripodi, Determining the spectrum of Meta $\left(K_{4}+e>K_{4}, \lambda\right)$ for any $\lambda$, Discrete Math. 308 (2008), 439-456.

[8] J. A. Kennedy, Maximum packings of $K_{n}$ with hexagons, Australas. J. Combin. 7 (1993), 101-110.

[9] T. P. Kirkman, On a problem in combinations, Cambridge and Dublin Math. J. 2 (1847), 191204.

[10] C. C. Lindner, G. Lo Faro, M. Meszka and A. Tripodi, Squashing maximum packings of $K_{n}$ with 8-cycles into maximum packings of $K_{n}$ with 4-cycles, Filomat 28:4 (2014), 887-896. 
[11] C. C. Lindner, M. Meszka and A. Rosa, From squashed 6-cycles to Steiner triple systems, J. Combin. Des. 22 (2014), 189-195.

[12] C. C. Lindner, K. T. Phelps and C. A. Rodger, The spectrum for 2-perfect 6-cycle systems, J. Combin. Theory Ser. A 57 (1991), 76-85.

[13] C. C. Lindner and C. A. Rodger, Design Theory, second ed., CRC Press, 2009.

[14] A. Rosa, On cycle decompositions of the complete graph into $(4 m+2)$-gons, Mat-fyz Časopis 16 (1966), 349-435.

[15] A. Rosa and C. Huang, Another class of balanced graph designs: balanced circuit designs, Discrete Math. 12 (1975), 269-293. 